\title{
SURFACE MODIFICATION CENTER
}

\author{
The MRS's Own Bill Appleton Heads ORNL's \\ Collaborative Research Center For Surface Modification
}

A collaborative research center, open to university and industry scientists for surface modification and advanced processing of materials to improve their performance characteristics, has been established at Oak Ridge National Laboratory.

The laboratory is one of four major energy-related facilities operated by Martin Marietta Energy Systems Inc. for the Department of Energy.

The research involves the use of high-powered lasers and particle accelerators to alter a few atomic layers at the surface of metals, ceramics, and semiconductors. Such modifications are designed to change physical characteristics in beneficial ways, including improved hardness and corrosion and wear resistance.

The modifications also can enhance electrical conductivity, enabling scientists to tailor the properties of semiconductors, for example, to particular electronic applications or devices-or to improve the efficiency and fabrication economy of solar cells.

The modified materials are of interest not only for these special applications, including longer-lived surgical implant alloys, but also for fundamental materials research, since the nonequilibrium fabrication processes lead to new and sometimes unique properties.

The Collaborative Research Center for Surface Modification, located in the Solid State Division of ORNL, now hosts some 40 academic and industrial groups and permits scientists to create new materials with equipment and techniques not usually available. It also allows industrial researchers to perform proof-of-principle experiments before large capital investments in particle accelerators and lasers are required.

MRS Vice President Bill R. Appleton, the Center's Director, notes: "Surface modifications may be the answer to many of today's high technology materials problems. Often, for example, it is necessary to choose a material for a particular application on the basis of its bulk properties, such as high strength and light weight. But a surface property, such as resistance to corrosion, may be totally unsuitable." Appleton also notes that surface modifications allow researchers selectively to alter a microthin layer on the surface to achieve a desired result. Through these techniques-ion implantation, ion mixing, and pulsed laser

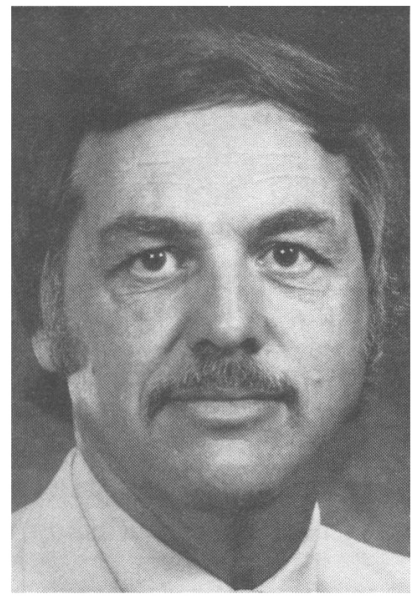

BILL APPLETON annealing-elements can be added in almost limitless ways to the atomic surface of a material.

In contrast, alloys normally are created from a molten solution of two or more metals, which slowly solidifies. In such conventional processes, physical laws limit the extent to which one metal will mix with another. Surface modification techniques, however, often can circumvent these considerations.

In ion implantation, atoms of elements with the desired characteristics-known as dopants-are accelerated to bombard and penetrate the surface of the target material,

\section{Proof-of-principle experiments possible Before large capital investments required}

altering the structure of the top few atomic layers. In ion beam mixing, ion bombardment is used to induce interactions between deposited thin films and the underlying material. Heat treating with extremely short, high-powered laser pulses melts and resolidifies the surface within a few billionths of a second, and this rapid solidification leads to new materials properties.

These techniques, which are used to some extent in the semiconductor industry to make computer chips, are not generally available to materials scientists. While not for commercial production or services, the Collaborative Research Center permits university and industrial researchers to work independently or with ORNL staff in demonstrating the feasibility of surface modification techniques for a particular application.

In the past, informal collaboration has resulted in significant materials advances. Recently, ORNL and University of Alabama at Birmingham scientists used ion implantation to alter the surface properties of artificial hip joint components made of titanium. By implanting nitrogen atoms on the outer skin of the titanium components, the surface becomes extremely resistant to wear and to body corrosion, thereby extending the lifespan of the artificial joint by more than 400 times, compared with currently available metal joints.

Materials scientists interested in collaborative research are invited to contact:

Bill R. Appleton

Director

Surface Modification and Characterization Facility

Oak Ridge National Laboratory

P.O. Box X

Oak Ridge, TN 37831

(615) 574-6283 